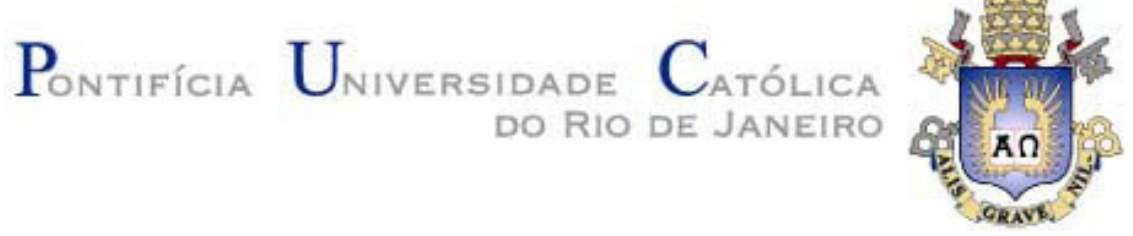

Renata Perlingeiro

As Implicações Estratégicas, no Nível da Empresa, das Alianças e Redes estabelecidas pela Embratel, Oi, Vivo e Tim, com vistas a contribuir à sua Vantagem Competitiva no Contexto Globalizado

Dissertação de Mestrado

Dissertação apresentada ao Programa de Pósgraduação em Administração de Empresas da PUCRio como requisito parcial para obtenção do título de Mestre em Administração de Empresas.

Orientadora: Prof ${ }^{\mathrm{a}}$. Teresia Diana Lewe van A. de Macedo-Soares

Rio de Janeiro

Setembro 2009 

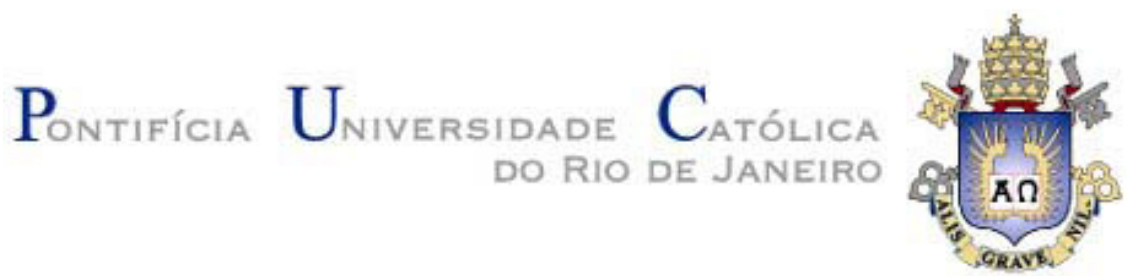

Renata Perlingeiro

As Implicações Estratégicas, no Nível da Empresa, das Alianças e Redes estabelecidas pela Embratel, Oi, Vivo e Tim, com vistas a contribuir à sua Vantagem Competitiva no Contexto Globalizado

Dissertação apresentada como requisito parcial para obtenção do grau de Mestre pelo Programa de Pósgraduação em Administração de Empresas da PUC-Rio. Aprovado pela Comissão Examinadora abaixo assinada.

Prof ${ }^{a}$. Teresia Diana Lewe van Aduard de Macedo-Soares Orientadora Departamento de Administração - PUC-Rio

Profa. Ana Heloísa Costa Lemos Departamento de Administração - PUC-Rio

Prof ${ }^{a}$. Irene Raguenet Trocolli FGV

Prof. Nizar Messari Vice-Decano de Pós-Graduação do CCS

Rio de Janeiro, 09 de setembro de 2009 
Todos os direitos reservados. É proibida a reprodução total ou parcial do trabalho sem autorização da universidade, da autora e da orientadora.

\section{Renata Perlingeiro}

Graduou-se em Administração de Empresas pela PUC-Rio em 2001. MBA em Gestão de Negócios pelo IBMEC - RJ. Trabalha na Oi , na área de Marketing.

Ficha Catalográfica

\section{Perlingeiro, Renata}

As implicações estratégicas, no nível da empresa, das alianças e redes estabelecidas pela Embratel, Oi, Vivo e Tim, com vistas a contribuir à sua vantagem competitiva no contexto globalizado / Renata Perlingeiro ; orientadora Teresia Diana Lewe van A. de Macedo-Soares . - 2009.

226 f. ; $30 \mathrm{~cm}$

Dissertação (Mestrado em Administração)Pontifícia Universidade Católica do Rio de Janeiro, Rio de Janeiro, 2009.

Inclui bibliografia

1. Administração - Teses. 2. Estratégia. 3. Alianças. 4. Telecomunicações. 5. Método de diferenças finitas. I. Macedo-Soares, Teresia Diana Lewe van A. de. II. Pontifícia Universidade Católica do Rio de Janeiro. Departamento de Administração. III. Título.

CDD: 658 


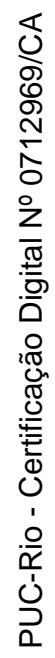

Para meus pais, Luiz e Tânia, pelo apoio e confiança. 


\section{Agradecimentos}

Aos meus pais, Tânia e Luiz, que sempre ressaltaram a importância do aprendizado contínuo e do desenvolvimento intelectual.

Ao meu marido, Rafael, pelo incansável apoio e paciência durante todo o processo de desenvolvimento deste trabalho, principalmente às vésperas do nosso casamento.

À minha orientadora, Prof ${ }^{a}$. T. Diana L. v. A de Macedo-Soares, pelas importantes contribuições que além de contribuir com o seu conhecimento, primou pelo rigor metodológico, o que foi fundamental para a qualidade desta pesquisa.

Aos professores que aceitaram participar da Comissão Examinadora desta pesquisa.

A todos os profissionais das empresas estudadas que contribuíram com a sua experiência para o sucesso deste estudo. 


\section{Resumo}

Perlingeiro, Renata; Macedo-Soares, Teresia Diana Lewe van Aduard de. As Implicações Estratégicas, no Nível da Empresa, das Alianças e Redes estabelecidas pela Embratel, Oi, Vivo e TIM, com vistas a contribuir à sua Vantagem Competitiva no Contexto Globalizado. Rio de Janeiro, 2009. 226p. Dissertação de Mestrado - Departamento de Administração, Pontifícia Universidade Católica do Rio de Janeiro.

A revolução tecnológica, as mudanças na estrutura competitiva da economia mundial e as necessidades financeiras motivaram vários países a realizar transformações estruturais no setor de telecomunicações. Essas mudanças incluíram a liberalização progressiva do setor, o estabelecimento de um órgão regulador e a privatização. Neste contexto, as empresas passaram a competir intensamente e buscar competências essenciais ao negócio. Esta busca quando realizada de forma isolada passou a exigir elevado esforço por parte das empresas. Com isso, o estabelecimento de alianças e redes na obtenção de vantagem competitiva se tornou uma realidade, que atualmente se apresenta com diversas características, conforme objetivos previamente definidos. Esta pesquisa tem por objetivo, avaliar as implicações estratégicas, no nível da empresa, das alianças e redes estabelecidas pela Embratel, Oi, Vivo e TIM, a fim de contribuir para a sua vantagem competitiva em um contexto globalizado. Para tanto, utilizou-se a metodologia de estudo de caso de Yin (2005), com a estratégia de triangulação dos métodos, que se baseia na investigação documental, no levantamento das percepções com a aplicação de questionários estruturados, além do levantamento de percepções complementares por intermédio de entrevistas. Os dados foram analisados com base no referencial teórico fundamentado na revisão da literatura, com auxílio de um levantamento documental e de um ferramental proposto: o modelo genérico integrativo de Macedo-Soares (2000) para a análise dos fatores organizacionais e o modelo SNA (MACEDO-SOARES, 2002) para a análise relacional. Constatou-se, através dos resultados obtidos, que as implicações estratégicas das alianças e redes agregam valor às análises tradicionais adicionando insights valiosos para a tomada de decisão no nível da empresa. O resultado desta análise resume-se no strategic fit, onde os fatores organizacionais 
capitalizam as forças, reduzem as fraquezas, de forma a explorar as oportunidades e minimizar as ameaças.

\section{Palavras-chave}

Estratégia; alianças estratégicas; telecomunicações 


\section{Abstract}

Perlingeiro, Renata; Macedo-Soares, Teresia Diana Lewe van Aduard de (Advisor). The strategic impacts, in the corporate level, of alliances and networks established by Embratel, Oi, Vivo and TIM, in order to contribute to their competitive advantage in a global environment. Rio de Janeiro, 2009. 226p. Msc. Dissertation - Departamento de Administração, Pontifícia Universidade Católica do Rio de Janeiro.

The technological revolution, along with the global economy structural changes and their financial constrains pushed several countries to perform profound reforms in the telecommunication industry. These changes included the industry gradual liberalization, the formation of a regulatory body and the privatization.Within this context, the companies struggled fiercely and went seeking core competencies to thrive in their competitive landscape. This isolated search led to an intense and costly effort made by the companies. In this sense, the formation of alliances and networks so as to obtain competitive advantages became a reality, which currently presents itself with several features, according to previously defined strategic goals. The main purpose of this research is to evaluate the strategic impacts, in the corporate level, of alliances and networks established by Embratel, Oi, Vivo and TIM, in order to contribute to their competitive advantage in a global environment. Thus, it was adopted the Yin (2005) case study methodology, with the strategy of methods triangulation, which is based on the documental investigation, on the perceptions raising applying structured questionnaires, as well as the complementary perceptions gathered through interviews. The data were analyzed based on the literature review of theoretical sources, aided by a documental gathering and by a set of proposed analytical tools: the integrated standard model of Macedo-Soares (2000) to analyze the organizational factors and the model SNA (MACEDO-SOARES, 2002) to relationship analysis. The conclusion drawn from the study was that the strategic alliances and networks analysis aggregate value to the traditional analysis adding valuable insights to the decision making in the corporate realm. 
The result of this research can be summarized on the strategic fit, where the organizational factors capitalize the strengths, reduce the weaknesses, thereby exploring the opportunities and minimizing the threats.

\section{Keywords}

Strategy; strategic alliances; telecommunications 


\section{Sumário}

1. Introdução 17

1.1. Questão Central da Pesquisa 21

1.2. Objetivos da Pesquisa $\quad 21$

1.2.1. Objetivo Maior 21

1.2.2. Questões Intermediárias 22

1.3. Relevância do Estudo 22

1.4. Delimitação do Estudo 23

1.5. Estrutura da Dissertação 24

2. Referencial Teórico 26

2.1. Revisão da Literatura 26

2.2. Fundamentação Teórica 33

2.2.1. Conceitos Centrais - Definições 33

2.2.2. Pressupostos 40

2.3. Ferramental Utilizado na Pesquisa 41

2.3.1. Ferramental Generic Integrative - GI

(MACEDO-SOARES, 200) para Análise Estratégica "Tradicional"

2.3.2. Macedo-Soares (2002) para análise relacional (SNA) 45

3. Metodologia de Pesquisa 55

3.1. Tipo de Pesquisa 55

3.2. Unidade de Análise e Sujeitos 60

3.2.1. Empresas Focais 60

3.3. Coleta de Dados 61

3.3.1. Pesquisa Bibliográfica/ Documental 62

3.3.2. Pesquisa Telematizada 62

3.3.3. Aplicação do Questionário 62

3.3.4. Levantamento Diferenciado Complementar - Entrevistas 64

3.3.5. Observação Participativa 64

3.4. Tratamento dos Dados 65

3.5. Limitações Metodológicas 66 
4. Resultados 69

4.1. O Mercado de Telecomunicações no Brasil 69

4.1.1. Histórico 69

4.1.2. Análise do Setor 71

4.1.3. O mercado de Telefonia Fixa 76

4.1.4. O mercado de Telefonia Móvel 78

4.2. Perfil das Empresas Estudadas 81

$\begin{array}{ll}\text { 4.2.1. Oi } & 81\end{array}$

4.2.1.1. Dados Gerais 81

4.2.1.2. Caracterização da Estratégia Atual da Oi 84

4.2.1.3. Os Fatores Organizacionais 87

4.2.1.4. Identificação e Avaliação das Implicações

Estratégicas das Alianças e Redes no Nível da Empresa 92

4.2.1.4.1. Identificação e Classificação das Alianças Estratégicas 92

4.2.1.4.2. As Principais Alianças com cada Parceiro 93

4.2.1.4.3. A Ego-Rede da Oi e suas Características 95

4.2.1.4.4. As Implicações Estratégicas, no Nível da Empresa, da Rede de Alianças formada pela Oi 97

4.2.1.4.5. Análise de Desempenho da Oi 101

4.2.2. TIM 103

4.2.2.1. Dados Gerais 103

4.2.2.2. Caracterização da Estratégia Atual da TIM 106

4.2.2.3. Os Fatores Organizacionais 109

4.2.24. Identificação e Avaliação das Implicações

Estratégicas das Alianças e Redes no Nível da Empresa 114

4.2.2.5. Identificação e Classificação das Alianças Estratégicas 114

4.2.2.6. Principais Alianças com cada Parceiro 114

4.2.2.6.1. A Ego-Rede da TIM e suas Características 116

4.2.2.6.2. As Implicações Estratégicas, no Nível da Empresa, da Rede de Alianças Formada pela TIM 117

4.2.2.6.3. Análise de Desempenho da TIM 122

4.2.3. Embratel 124

4.2.3.1. Dados Gerais 124 
4.2.3.2. Caracterização da Estratégia Atual da Embratel 126

4.2.3.3. Os Fatores Organizacionais 128

4.2.3.4. Identificação e Avaliação das Implicações

Estratégicas das Alianças e Redes no Nível da Empresa 133

4.2.3.4.1. Identificação e Classificação das Alianças Estratégicas 133

4.2.3.4.2. As Principais Alianças com cada Parceiro 133

4.2.3.4.3. A Ego-Rede da Embratel e suas Características 135

4.2.3.4.4. As Implicações Estratégicas, no Nível da Empresa, da Rede de Alianças formada pela Embratel 137

4.2.3.4.5. Análise de Desempenho de Embratel 141

4.2.4. Vivo 142

4.2.4.1. Dados Gerais 142

4.2.4.2. Caracterização da Estratégia Atual da Vivo 144

4.2.4.3. Os Fatores Organizacionais 146

4.2.4.4. Identificação e Avaliação das Implicações

Estratégicas das Alianças e Redes no Nível da Empresa 151

4.2.4.4.1. Identificação e Classificação das Alianças Estratégicas 151

4.2.4.4.2. As Principais Alianças com cada Parceiro 151

4.2.4.4.3. A Ego-Rede da Vivo e suas Características 152

4.2.4.4.4. As Implicações Estratégicas, no Nível da Empresa, da Rede de Alianças formada pela Vivo 155

4.2.4.4.5. Análise de Desempenho da Vivo 160

5. Discussão dos Resultados 162

5.1. Impacto das Implicações Estratégicas Relacionais em Termo de

Forças e Fraquezas 162

6. Conclusões 169

7. Referenciais Bibliográficas 172

8. Anexos 177 


\section{Lista de figuras}

Figura 1 - Elementos Comuns em Estratégias de

Sucesso - Gant (2006)

Figura 2 - Modelo Genérico Integrativo - GI

(Macedo Soares, 2000) 43

Figura 3 - Modelo SNA (Macedo - Soares,2002) 49

Figura 4 - Fases da Pesquisa $\quad 57$

Figura 5 - Distribuição de Cargo por Respondentes

da Pesquisa

Figura 6 - Metodologia para o Desenvolvimento e

Avaliação de Questionário - Macedo-Soares \& Neves (2002) 63

Figura 7 - Triangulação dos Métodos - Yin (2005) 68

Figura 8 - Comparativo dos Impostos nos Principais

Países do Mundo $\quad 74$

Figura 9 - Principais Grupos de Telecom Atuantes no Brasil 75

Figura 10 - Evolução Anual do Número de Acessos Fixos

no Brasil

77

Figura 11 - Quantidade de Telefones Fixos nos

Principais Mercados do Mundo

78

Figura 12 - Existem no Brasil 7 Grupos de Operadoras

de Celular

79

Figura 13 - Desempenho Operacional das Operadoras

Móveis

Figura 14 - Quantidade de Celulares no Mundo

Figura 15 - Principais Mercados de Celular no Mundo

Figura 16 - Área de Cobertura da Oi

Figura 17 - Participação acionária da Oi

Figura 18 - Principais Parceiros da Oi

Figura 19 - Fatores Determinantes para o

Estabelecimento de Alianças Estratégicas

Figura 20 - Ego-Rede da Oi 96

Figura 21 - Percepção de Centralidade 
Figura 22 - Classificação do Grau de Dificuldade

para o Acesso aos Recursos-Chave dos Parceiros

Figura 23 - Critérios Utilizados pela OI para

Buscar a Compatibilidade entre ela Própria e seus Parceiros

Figura 24 - Área de Cobertura da TIM

104

Figura 25 - Participação Acionária da TIM

105

Figura 26 - A Estratégia Adotada pela TIM

108

Figura 27 - Principais Atores-Chave da TIM

Figura 28 - Ego-Rede de TIM

Figura 29 - Percepção de Centralidade

Figura 30 - Critérios Utilizados pela TIM para Buscar

a Compatibilidade entre ela Própria e seus Parceiros

122

Figura 31 - Ego-Rede da Embratel

Figura 32 - Indicador para Medir o Sucesso das

Alianças no Desempenho Global da Empresa

Figura 33 - Critérios Utilizados pela Embratel para Buscar

a Compatibilidade entre ela Própria e seus Parceiros

Figura 34 - Estrutura Acionária da Vivo

Figura 35 - Área de Cobertura da Vivo

Figura 36 - A Ego-Rede da Vivo

Figura 37 - Indicador para Medir o Sucesso das Alianças

no Desempenho Global da Empresa

Figura 38 - Critérios Utilizados pela Vivo para Buscar

a Compatibilidade entre ela Própria e seus Parceiros

Figura 39 - Comparação Gráfica das Análises "Tradicional"

e "Relacional" 


\section{Lista de quadros}

Quadro 1 - Comparativo de Paradigmas entre Parcerias Convencionais e Alianças Estratégicas de Acordo

com Doz \& Hamel (1998)

Quadro 2 - Tipos de Alianças Estratégicas - Gouvêa

(2008) - readaptado para a indústria de telecomunicações

Quadro 3 - Lista dos Atributos Necessários/ Desejáveis para cada Variável Principal Independente, para a Gestão

Estratégica Orientada aos Clientes Adaptados para o Setor de Telecomunicações

Quadro 4-C Constructos para análise relacional - nível

da empresa focal (Macedo - Soares, 2002)

Quadro 5 - Constructos para a análise relacional - nível

corporativo da empresa focal

Quadro 6 - Composição dos indicadores de desempenho

para a avaliação das empresas do setor de telecomunicações

Quadro 7 - Fatores Organizacionais e suas Implicações

Estratégicas, no sentido de Constituírem Forças e

Fraquezas - Oi

Quadro 8 - Principais Características das Alianças

entre a Oi e seus Principais Parceiros

Quadro 9 - Implicações Estratégicas da Ego-Rede da Oi,

no Nível da Empresa

Quadro 10 - Indicadores de Desempenho da Oi

Quadro 11 - Fatores Organizacionais e suas

Implicações Estratégicas, no sentido de Constituírem

Forças e Fraquezas - TIM

Quadro 12 - Principais Características das Alianças

entre a TIM e seus Principais Parceiros

Quadro 13 - Implicações Estratégicas da Ego-Rede da TIM, no Nível da Empresa 
Quadro 15 - Fatores Organizacionais e suas

Implicações Estratégicas, no sentido de Constituírem

Forças e Fraquezas - Gouvêa (2008)

Quadro 16 - Principais Características das Alianças

entre a Embratel e seus Principais Parceiros

136

Quadro 17 - Implicações Estratégicas da Ego-Rede

da Embratel, no Nível da Empresa

Quadro 18 - Indicadores de Desempenho da Embratel

Quadro 19 - Fatores Organizacionais e suas Implicações

Estratégicas, no sentido de Constituírem Forças e

Fraquezas - Vivo

Quadro 20 - Principais Características das Alianças entre

a Vivo e seus Principais Parceiros

Quadro 21 - Implicações Estratégicas da Ego-Rede da Vivo, no Nível da Empresa

Quadro 22 - Indicadores de Desempenho da Vivo

Quadro 23 - Confronto de Implicações Estratégicas -

Análise Tradicional versus Relacional 